This document was prepared in conjunction with work accomplished under Contract No. DE-AC09-96SR18500 with the U.S. Department of Energy.

This work was prepared under an agreement with and funded by the U.S. Government. Neither the U. S. Government or its employees, nor any of its contractors, subcontractors or their employees, makes any express or implied: 1 . warranty or assumes any legal liability for the accuracy, completeness, or for the use or results of such use of any information, product, or process disclosed; or 2 . representation that such use or results of such use would not infringe privately owned rights; or 3 . endorsement or recommendation of any specifically identified commercial product, process, or service. Any views and opinions of authors expressed in this work do not necessarily state or reflect those of the United States Government, or its contractors, or subcontractors. 
WSRC-MS-2006-00395

\title{
SYNCHROTRON X-RAY BASED CHARACTERIZATION OF CdZnTe CRYSTALS
}

\author{
Martine C. Duff, Douglas B. Hunter, Patterson Nuessle \\ Savannah River National Laboratory (SRNL), Aiken, SC
}

David R. Black, Harold Burdette, Joseph Woicik

National Inst. of Standards and Technology (NIST), Gaithersburg, MD

Arnold Burger and Michael Groza

Fisk University, Nashville, TN

* Corresponding author's phone: (803)725-2054; fax: (803)725-4704, e-mail:

martine.duff@srnl.doe.gov.

\begin{abstract}
Synthetic CdZnTe or "CZT" crystals can be used for the room temperature-based detection of $\gamma$-radiation. Structural/morphological heterogeneities within CZT, such as twinning, inclusions, and polycrystallinity can affect detector performance. We used a synchrotron-based X-ray technique, specifically extended X-ray absorption fine-structure (EXAFS) spectroscopy, to determine whether there are differences on a local structural level between intact CZT of high and low radiation detector performance. These studies were complemented by data on radiation detector performance and transmission IR imaging. The EXAFS studies revealed no detectable local structural differences between the two types of CZT materials.
\end{abstract}

Key words: EXAFS, IR Imaging, radiation detectors, semiconductors. 
WSRC-MS-2006-00395 


\section{Introduction}

Synthetic CdZnTe or "CZT" crystals can be used for the room temperature-based detection of gamma radiation. However, the radiation detection properties of CZT crystals vary widely. These variances are not well understood but they are attributed to structural and morphological heterogeneities within the crystals, such as twinning, secondary phases and polycrystallinity. Such heterogeneities within the CZT can often result in poor $\gamma$-ray detection performance because they lead to poor peak resolution and peak tailing.

The average bulk structural properties of CdZnTe materials have been characterized on an atomic level using synchrotron X-ray techniques by several researchers. ${ }^{1,2,3,4}$ The ternary Cd-Zn-Te system forms a cubic zinc blende structure. Two previous EXAFS studies investigated the local structure of $\mathrm{Cd}_{1-\mathrm{x}} \mathrm{Zn} \mathrm{n}_{\mathrm{x}} \mathrm{Te}$ for $\mathrm{x}=0$ to $\mathrm{x}=1.0$. These EXAFS studies were conducted with powdered samples and determined that the bond lengths between constituent atoms deviate only slightly from the binary forms of $\mathrm{ZnTe}$ and $\mathrm{CdTe}$. The current study was aimed at conducting EXAFS with intact (not ground) single-crystals that consisted of a fixed $\mathrm{Zn}$ doping in the structure but varied in their electronic properties as radiation detectors. This study presents data on intact CZT crystals that have known X-ray detector performance (specifically, poor and good). Other characterization data on these two types of materials such as resistivity, FT-IR imaging, and electron mobility are presented.

\section{Methods and Materials}

The CZT crystals (namely the host single crystals called CZT1 and CZT2) that we examined were made according to the Modified Vertical Bridgman (MVB) method to have 10\% $\mathrm{Zn}$ content as described in Li et al. $(2001)^{5}$ and procured from Yinnel Tech (South Bend, 
$(N)^{*}$. Due to the variety of experimental studies planned for these two crystals, multiple sections had to be used, rather than compare results directly from the same section. The crystals for the first set of characterization studies (CZT1a and CZT2a) were $5 \times 7 \times 2.3 \mathrm{~mm}^{3}$ and $10.5 \times 10.5 \times 3.44 \mathrm{~mm}^{3}$ in size (respectively). These crystals were polished and then characterized by IR imaging using a CCD camera for image recording, and prepared with gold $(\mathrm{Au})$ contacts deposited by the sputtering technique. The Au contact allowed for detector performance testing, lifetime mobility measurements and measurements of mobility-lifetime product. We used a second set of sister CZT materials (namely CZT1b and CZT2b) for the XAFS studies. This was required because the Au surface contacts used for radiation performance testing would interfere with the EXAFS studies and the crystals were much too thick. Therefore, we required the use of sister thin section $(\sim 100-\mu \mathrm{m}$ thick) materials of these crystals for our characterization studies with EXAFS spectroscopic techniques.

Preliminary EXAFS spectroscopic studies were performed at the Advanced Photon Source (APS) at Argonne National Laboratory (Argonne, IL) using Basic Energy Sciences Synchrotron Research Collaborative (BESSRC) bending magnet-12 (data not shown). Follow-up studies were conducted at NIST beamline bending magnet X23a2 at the National Synchrotron Light Source (NSLS) at Brookhaven National Laboratory (Upton, NY). The EXAFS studies were conducted at three different elemental absorption edge energies (Cd Kedge at $26.711 \mathrm{keV}$, Te K-edge at $31.814 \mathrm{keV}$ and $\mathrm{Zn} \mathrm{K-edge} \mathrm{at} 9.659 \mathrm{keV})$. The Cd- and Te-EXAFS studies were performed in transmission using Ar-gas filled ion chambers. The ZnEXAFS studies were performed in transmission (using $\mathrm{N}_{2}$-gas filled ion chambers) and in fluorescence mode using a Lytle Detector with Ge filter (3 absorbance unit lengths). 
The background contribution to the EXAFS spectra was removed using an algorithm (AUTOBK) developed by Newville et al. $(1993)^{6}$, which minimizes R-space values in low $k$ space. Each chi data set was read into the WINXAS analysis package. ${ }^{7}$ Replicate EXAFS scans were co-added to improve signal to noise. Due to numerous diffraction peaks in the Zn-EXAFS spectra, the samples were spun (rotation axis normal to the incident X-ray beam) to average out the diffraction peaks in the EXAFS spectra. All EXAFS spectra were collected to $850 \mathrm{eV}$ beyond the K-edges for $\mathrm{Cd}, \mathrm{Zn}$ and Te. All EXAFS data were analyzed for wave vectors $(k)$ from 2 to $13 \AA^{-1}$. The chi data were $k^{2}$-weighted and Fourier-transformed to yield R-space data. ${ }^{8}$ Simulated EXAFS spectra were also generated based on the documented crystallographic properties for $\mathrm{Cd}, \mathrm{Zn}$ and Te using ab initio based theory, which involved FEFF 8.2 a program created by researchers at the Univ. of Washington. ${ }^{9}, 10,11,12,13$ Model fits were performed in R-space. A cleaved high purity Te metal ingot of approximately $80-\mu \mathrm{m}$ in thickness was used as a reference compound for Te-EXAFS on the CZT crystals. The EXAFS data were fit against a known crystal structure for Te metal (Cherin and Unger, 1967). ${ }^{14}$ Crystal structures for CZT were taken from Wkchoff (1963). ${ }^{15}$ 


\section{$\underline{\text { Results }}$}

Figures $1 \mathrm{~A}-\mathrm{E}$ and $2 \mathrm{~A}-\mathrm{D}$ show data for resistivity measurements, transmission-based IR images, radiation detector performance testing and mobility $(\mu)$-lifetime $(\tau)$ product for CZT1a and CZT2a materials respectively. Both CZT materials had high resistivity measurements $(\rho)$ with CZT1a having $\rho=1.5 \times 10^{11} \Omega \mathrm{cm}$ and CZT2a having a resistivity $\rho=$ $1.5 \times 10^{11} \Omega \mathrm{cm}$. The IR imaging studies for the sister thin sections to those from the EXAFS studies indicate that there are also notable differences between these two materials with respect to secondary phase distribution and size. Secondary phase "Te inclusions" are defined in this paper and elsewhere as being 1 to $50 \mu \mathrm{m}$ in diameter and objects of this size are detectable using transmission $I R .^{16}$ Objects of smaller size than $1 \mu \mathrm{m}$ are referred to as "Te precipitates" by Rudolph and Mühlberg (1993) and in CdTe crystals are in the 10 to 30 $\mathrm{nm}$ range which are not detectable by IR. ${ }^{16}$ For example, if the definitions of Rudolph and Mühlberg (1993) are used, Fig. 2B contains an IR image for the poor performing crystal which appears to have numerous small precipitates—although these particles are difficult to image because the spatial resolution of IR is near one micron. In contrast, Fig. 1B shows an IR image for the good performing CZT crystal (as shown in Fig. 1B) that has numerous inclusions that are much larger in size than that shown in Fig. 2A. These studies indicate that there is a possible relationship between secondary phase size and distribution and radiation detector performance as observed by others previously (Carini et al., 2006). ${ }^{4}$ Radiation detector performance was evaluated using a gamma and alpha source of americium-241. Fig. 1B shows a spectrum obtained from the first CZT crystal that had high resolution and limited peak tailing—indicative of a good performing crystal. In contrast, very poor alpha particle spectral resolution was obtained from the other crystal (CZT2a) as shown in Fig. 2C. 
Due to the low signal to noise and poor quality of this material, gamma-ray measurements were not possible. Measurements of mobility $(\mu$ )-lifetime $(\tau)$ product for CZT2a (shown in Fig. $1 \mathrm{E})$ indicate a high $\mu \tau$ product of $5 \times 10^{-3} \mathrm{~cm} / \mathrm{V}$ for the good performing CZT material. Measurements of mobility lifetime product were not possible with the poor performing crystal. With the results from these studies, we selected these two types of materials (poor and high quality detector grade) for further study using EXAFS spectroscopic techniques.

The EXAFS data for Te in Te reference metal agree well in radial distance $(R(\AA))$ and coordination number $(\mathrm{CN})$ with the crystallographic literature within less than $\pm 0.01 \AA$. EXAFS spectroscopic techniques were used to characterize the local coordination and radial distance environment of the $\mathrm{Cd}, \mathrm{Zn}$, and Te in the two CZT sister materials (CZT1b and $\mathrm{CZT} 2 \mathrm{~b}$ ) from the previously discussed IR and detector performance studies. Table 1 shows the results of our fits for first and second shell neighbors according to the $\mathrm{Cd}-, \mathrm{Te}-$, and $\mathrm{Zn}$ EXAFS studies. Pseudo radial distribution function plots of the EXAFS data for all three absorption edges are shown in Fig. 3.

Acquisition of EXAFS data for all three atoms for the two crystals is presented in Table 1. The nearest neighbor bond lengths were consistent with previous publications for $10 \% \mathrm{Zn}$ in CdZnTe ${ }^{1,3}$ Comparison of $\mathrm{Cd}-\mathrm{Te}$ and Te-Cd radial distances both within the same crystal and between crystals does not reveal any statistically significant differences within the uncertainty of the measurements $( \pm 0.01 \AA)$.

Comparison of the Zn X-ray absorption near edge structure (or XANES, data not shown) portion of the $\mathrm{Zn}$ absorption data in transmission and fluorescence indicated that selfabsorption was not an issue with these samples. Severe diffraction glitches were observed in the EXAFS spectra at the $\mathrm{Zn}$ absorption edge energy. The crystals were rapidly spun to 
average the diffraction orientations across the EXAFS energy scan and fluorescence data were collected. The same observation is made for $\mathrm{Zn}-\mathrm{Te}$ and Te-Zn EXAFS data as was made for the Cd-Te and Te-Cd interactions. There were no significant differences between the two CZT crystals.

The fits for the Debye-Waller factor $\left(\sigma^{2}\right)$ which is a measure of disorder in the crystal is also the same between the two types of crystals for each atom of interest (Table 1). In summary, high quality EXAFS spectra were collected on crystals that exhibited markedly different qualities in their electronic properties as X-ray detectors.

\section{Discussion}

The data in this study suggest that it is not only the CZT structure that provides functionality as a semi-conducting radiation detector but other features like secondary phase size and distribution are important factors that effect performance (either directly or indirectly). Several studies on CZT detector performance using collimated gamma ray sources and various types of detector fabrications have attributed poor performance to regions of CZT and CdTe that are rich in Te secondary phases. ${ }^{17,18,19}$ These and other studies concluded that these Te-rich areas limit the collection and mobility of electrons (through trapping) and that this factor strongly limits performance in those materials that are being produced with fairly current methodologies. ${ }^{17}$

Some researchers have classified these secondary phases as two types of Te-rich phases that form based on processes that take place during crystal growth and cooling. ${ }^{16}$ The first type of Te-rich phase is the Te inclusions, which are thought to form in melts with excess Te at the solid-liquid interface (in the absence of control over melt composition during 
synthesis). These phases range from 1 to several tens of microns in size. The second of these Te-rich phases (called precipitates) are thought to form during cool-down from the retrograde slope portion of the solidus. These precipitates are much smaller in size ranging from 10 to $30 \mathrm{~nm}$.

Because IR imaging has been traditionally used to detect secondary phases in CZT and it is limited spatially to detectable objects greater than $1 \mu \mathrm{m}$ in diameter, there is an absence of published information about smaller-sized secondary phases in CZT and their effect on detector performance. Without sufficient information to attribute detector performance to secondary phase size distribution and concentration, it is difficult to determine the relationship between the secondary phases and the electronic properties of CZT. It is possible that there may be a continuum of secondary phase particle size within CZT that remain to be insufficiently detected with modern analytical techniques. A spatial effect on performance due to these secondary phases is to be expected as studies have shown with collimated beams. ${ }^{18}$ However, these secondary phases appear to have a small contribution to the total mass and do not have a negative effect on bulk detector performance in these studies.

The EXAFS data for the two types of performing materials indicate that there are no significant differences in the local atomic structure of the three primary elements that comprise this highly crystalline material. Regardless of the demonstrated differences in detector performance, precipitate concentration/distribution and resistivity, these two types of CZT material are nearly indistinguishable using EXAFS spectroscopic techniques.

In summary, these studies indicate that radiation detector performance is related to not only the highly crystalline semi-conducting CdZnTe structure but to other factors as well, such 
as the presence of secondary phases. Even though secondary phases $(>1 \mu \mathrm{m})$ are known to limit local electron mobility, their presence is not sufficient to degrade bulk detector performance in these studies.

${ }^{*}$ Certain commercial equipment, instruments, or materials are identified in this paper in order to specify the experimental procedure adequately. Such identification is not intended to imply recommendation or endorsement by the National Institute of Standards and Technology, nor is it intended to imply that the materials or equipment identified are necessarily the best available for the purpose. 


\section{$\underline{\text { References }}$}

1) Motta, N., Balzarottie, A., Letardi, P., Kisiel, A., Czyzyk, M.T., Zimnal-Starnawska, M., and M. Podgorny. (1985). Random distribution and miscibility of $\mathrm{Cd}_{1-\mathrm{x}} \mathrm{Zn} \mathrm{n}_{\mathrm{x}} \mathrm{Te}$ alloy from EXAFS. J. Crystal Growth 72:205-209.

2) Goorsky, M.S., Yoon, H., Ohler, M., and K. Liss. (1999). Defect depth profiling of CdZnTe using high-energy diffraction measurements. SPIE. 3768:108-114.

3) Koteski, V., Hass, H., Holub-Frappe, E., Ivanovic, N., and H.,-E. Mahnke. (2004). Bond lengths in $\mathrm{Cd}_{1-\mathrm{x}} \mathrm{Zn}_{\mathrm{x}} \mathrm{Te}$ beyond linear laws revisited. J. Alloys Compounds 371:138-141. 4) Carini, G.A., Bolotnikov, A.E., Camarda, G.S., Wright, G.W., Li, L., and R.B. James. (2006). Effect of Te precipitates on the performance of CdZnTe detectors. Appl. Phys. Lett. 88:143515.

5) Li, L., Lu, F., Shah, K., Squillante, M., Cirinano, L., Yao, W., Olson, R.W., Luke, P., Nemirovsky, Y., Burger, A., Wright, G., and R.B. James. (2001). Nuclear Science Symposium Conference Record IEEE 4:2396-2400.

6) Ressler, T. (1999). WinXAS. A Program for X-ray Absorption Spectroscopy Data Analysis under MS Windows.

7) Newville, M., Livins, P., Yacoby, Y., Rehr, J.J., and Stern, E.A. (1993). Near-edge X-rayabsorption fine-structure of $\mathrm{Pb}-\mathrm{A}$ comparison of theory and experiment. Phys. Rev. B-Cond. Matter, 47:14126-14131.

8) Sayers, D.E. and Bunker, B.A. (1988). In X-ray Absorption: Techniques of EXAFS, SEXAFS and XANES. Koningsberger, D.C. and Prins, R. (eds). Wiley, New York, Chap. 6. 9) Mustre de Leon, J., Rehr, J.J., Zabinsky, S.I., and Albers, R.C. (1991). Ab initio curvedwave x-ray-absorption fine structure, Phys. Rev. B44:4146. 
10) Rehr, J.J. and Albers, R.C. (1990). Scattering-matrix formulation of curved-wave multiple-scattering theory: Application to x-ray-absorption fine structure. Phys. Rev.

B41:8139.

11) Rehr, J.J., Mustre de Leon, J., Zabinsky, S.I., and Albers, R.C. (1991). Theoretical X-ray absorption fine structure standards. J. Am. Chem. Soc. 113:5135.

12) Rehr, J.J., Zabinsky, S.I., and Albers, R.C. (1992). High-order multiple scattering calculations of x-ray-absorption fine structure. Phys. Rev. Let. 69:3397.

13) Stern, E.A., Newville, M., Ravel, B., Yacoby, Y. and Haskel, D. (1995). The UWAFS analysis package - Philosophy and details. Physica B. 208-209:117-120.

14) Cherin, P.C. and P. Unger. (1967). Two-dimensional refinement of the crystal structure of tellurium. Acta Crystallographica 23:670-671.

15) Wkchoff, R.W.G. (1963). Crystal Structures 1. Second Ed. Wiley Interscience Publishers, NY, NY.

16) Rudolph, P. and M. Mühlberg. (1993). Basic problems of vertical Bridgman growth of CdTe. Materials Science and Engineering B16:8-16.

17) Szeles, C., Cameron, S. E., Ndap, J.-O., and W.C. Chalmers. (2002). Advances in the crystal growth of semi-insulating CdZnTe for radiation detector applications. IEEE Trans. Nucl. Sci. 49:2535-2540.

18) Luke, P.N., Amman, M., and J.S. Lee. (2004). Factors affecting energy resolution of coplanar-grid CdZnTe detectors. IEEE Trans. Nucl. Sci. 51:1199-1203.

19) Parker, B.H., Stahle, C.M., Barthelmy, S.D., Parsons, A.M., Tueller, J., Van Sant, J.T., Munoz, B.F., Snodgrass, S.J., and R.E. Mullinix. (1999). Correlation between bulk material 
defects and spectroscopic response in cadmium zinc tellurium detectors. SPIE 3768:129137.

\section{Acknowledgements}

This work is supported by the National Nuclear Security Administration's Office of Research and Development (NA-22). Use of the APS was supported by the U.S. Department of Energy, Office of Science, Office of Basic Energy Sciences, under Contract No. W-31-109ENG-38. Use of the NSLS, Brookhaven National Laboratory, was supported by the U.S. Department of Energy, Office of Science, Office of Basic Energy Sciences, under Contract No. DE-AC02-98CH10886. We acknowledge Dr. Nadia Leyarovska of BESRRC at APS for her excellent assistance at BM-12. The authors at Fisk University gratefully acknowledge financial support from NSF for Center of Research Excellence in Science and Technology (CREST) grant no. 0420516, and from US DOE Office of Nonproliferation Research and Engineering (NA-22), Grant No. DE-FG52-05NA27035. 
A)

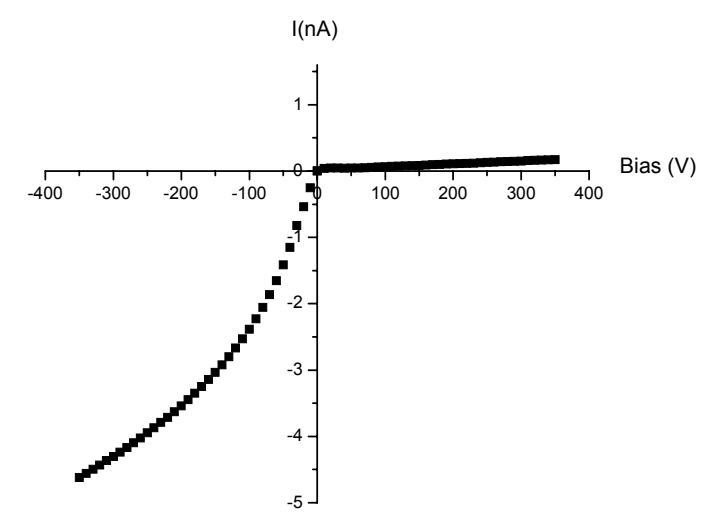

C)

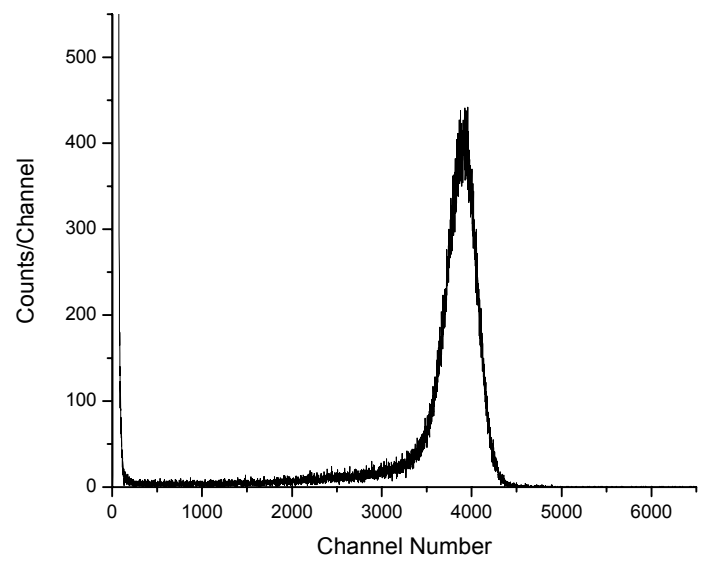

B)

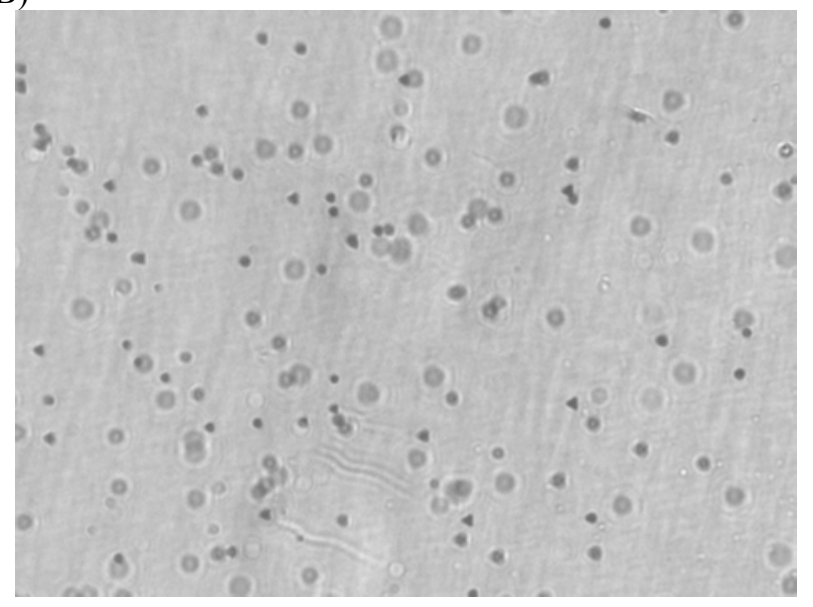

D)

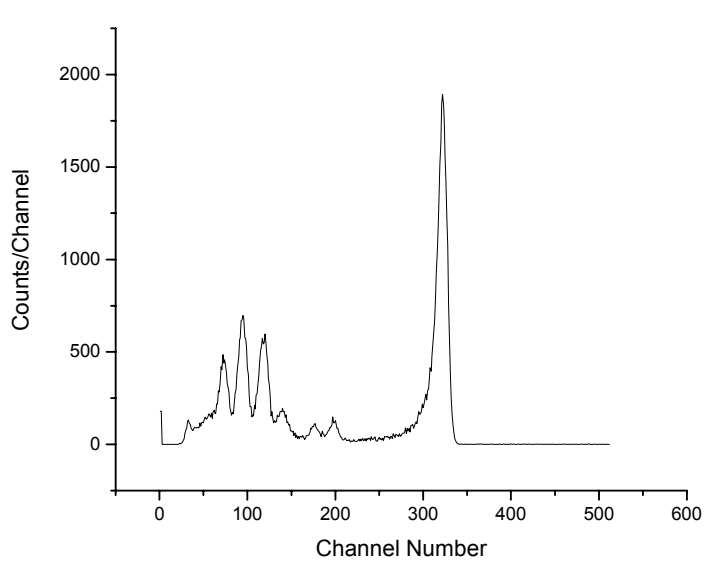

E)

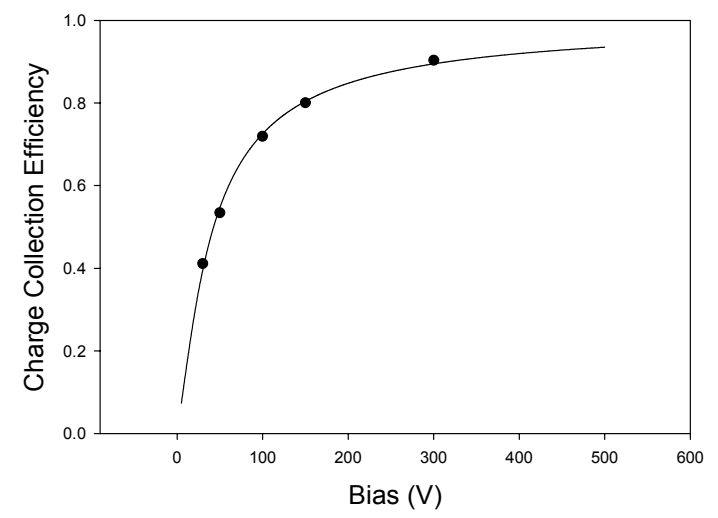

Figure 1. Detector grade CZT sample called CZT1a. A) Resistivity measurements. B) Transmission IR image (Area: $1 \times 1.5 \mathrm{~mm}^{2}$; depth of field is $0.25 \mathrm{~mm}$ ). C) Alpha particle measurement using Am-241 (at a bias of $500 \mathrm{~V}$ with a FWHM of 9.5\%). D) Gamma-ray measurement using Am-241 (at a bias of 500V and a FWHM of 4.1\%), and E) Mobility-lifetime product measurement: $\mu \tau=5 \times 10^{-3} \mathrm{~cm}^{2} / \mathrm{V}$. 
A)

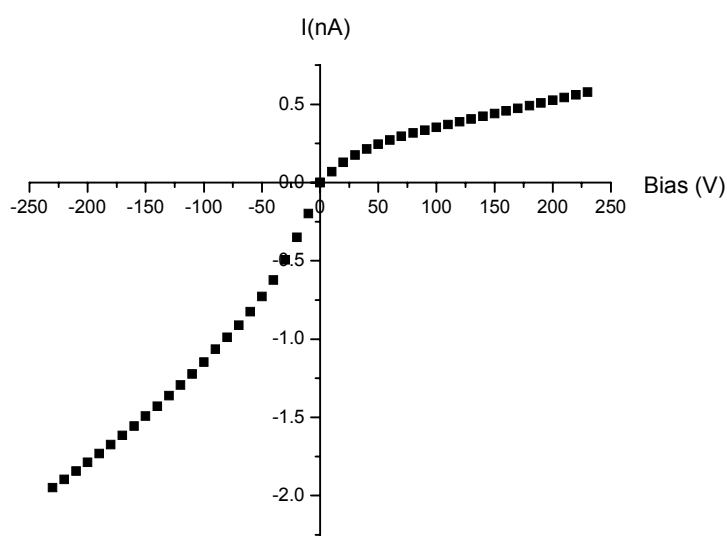

C)

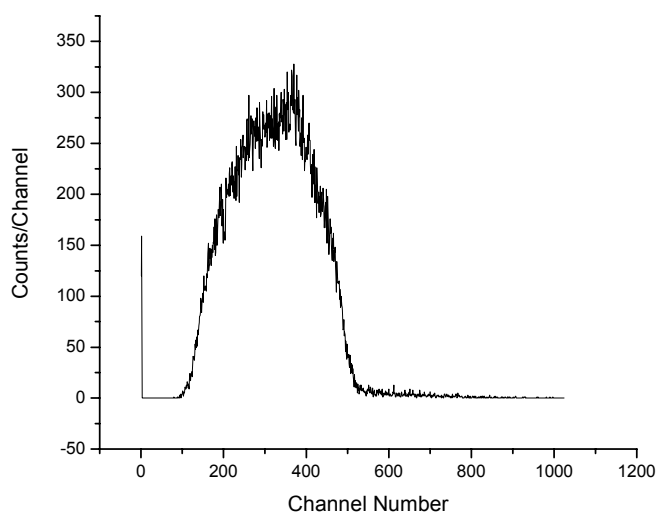

B)

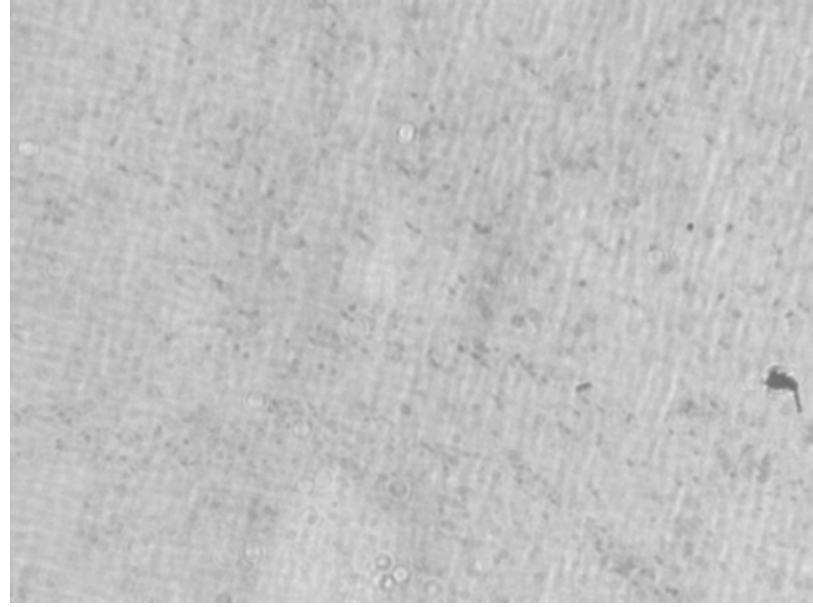

Figure 2. Non-detector grade CZT sample called CZT2a. A) Resistivity measurements. B) Transmission IR image (Area: $1 \times 1.5 \mathrm{~mm}^{2}$; depth of field is $0.25 \mathrm{~mm}$ ). C) Alpha particle measurement using Am-241 (at a bias of $500 \mathrm{~V}$ with a FWHM of $94 \%$ ). Due to the low signal to noise and poor quality of this material, gamma-ray measurements and mobility-lifetime product determinations were not possible. 


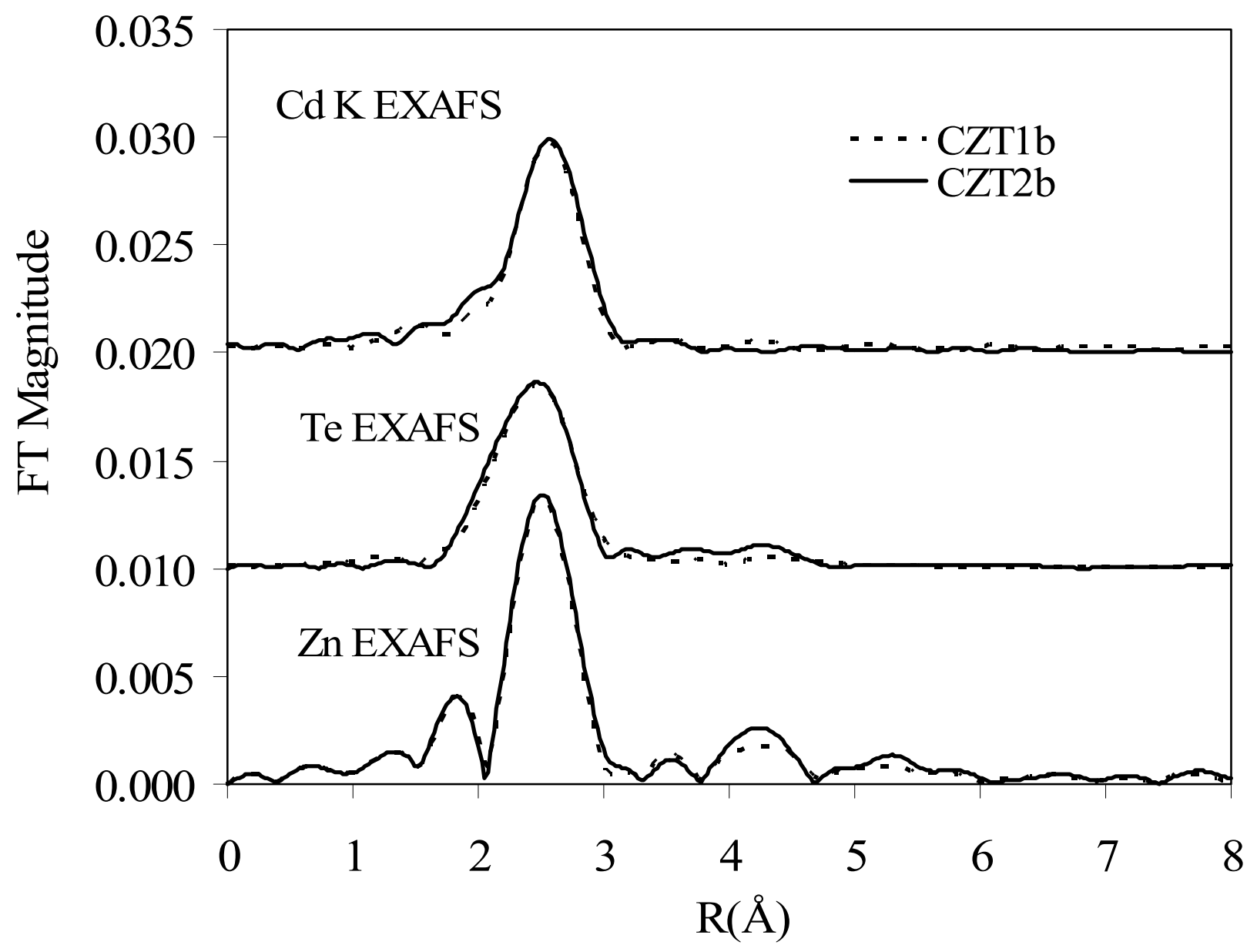

Figure 3. Pseudo radial distribution function plots of the EXAFS data for all three absorption edges for CZT1b and CZT2b. 
Table 1. The results of the EXAFS data fits for first coordination shell atom interactions in Te metal and the two types of CZT.

\begin{tabular}{|c|c|c|c|c|c|c|}
\hline Material & Interaction & $\mathbf{S}^{2}$ * & $\mathrm{CN}^{* *}$ & $\mathbf{R}(\AA)^{* * *}$ & $\sigma^{2}$ & $E_{0}(e V)$ \\
\hline \multirow[t]{2}{*}{ Te Metal } & Te-Te & 0.94 & 2 & 2.83 & 0.005 & 6.7 \\
\hline & Te-Te & 0.94 & 4 & 3.50 & 0.019 & 6.7 \\
\hline CZT1b & $\mathrm{Zn}-\mathrm{Te}$ & 0.94 & 4 & 2.66 & 0.007 & 3.5 \\
\hline $\mathrm{CZT} 2 \mathrm{~b}$ & $\mathrm{Zn}-\mathrm{Te}$ & 0.94 & 4 & 2.67 & 0.007 & 3.5 \\
\hline CZT1b & Cd-Te & 0.94 & 4 & 2.78 & 0.006 & 3.3 \\
\hline $\mathrm{CZT} 2 \mathrm{~b}$ & Cd-Te & 0.94 & 4 & 2.80 & 0.006 & 6.5 \\
\hline \multirow[t]{2}{*}{ CZT1b } & Te-Cd & 0.94 & 4 & 2.80 & 0.008 & 6.1 \\
\hline & Te-Zn & 0.94 & 1 & 2.67 & 0.003 & 6.1 \\
\hline \multirow[t]{2}{*}{$\mathrm{CZT} 2 \mathrm{~b}$} & Te-Cd & 0.94 & 4 & 2.79 & 0.006 & 3.2 \\
\hline & Te-Zn & 0.94 & 1 & 2.70 & 0.003 & 3.2 \\
\hline
\end{tabular}

* $\mathrm{S}^{2}$ was determined from the Te metal standard by fixing the known CN. This value of $\mathrm{S}^{2}$ was then used as a fixed parameter in the CZT fits. 
${ }^{* *} \mathrm{CN}$ was fixed for all CZT analyzes. Allowing $\mathrm{CN}$ to float during fitting resulted in less than $1 \%$ change from the fixed value. In the case of Te where 2 sub-shells were fit ( $\mathrm{Te}-\mathrm{Cd}$ and Te-Zn), the contribution from each shell was waited as Te-Cd $90 \%$ and $\mathrm{Te}-\mathrm{Zn}$ as $10 \%$. ${ }^{* * *} R(\AA), \sigma^{2}$ and $E_{0}$ were not constrained during fitting. 